\title{
Capacitive seat sensors for multiple occupancy detection using a low-cost setup
}

\author{
Adriaan S. Zeeman and Marthinus J. Booysen \\ Department of Electrical and Electronic Engineering \\ Stellenbosch University \\ Stellenbosch, South Africa \\ Email: ad.zeeman@gmail.com,mjbooysen@sun.ac.za
}

\author{
Giuseppe Ruggeri and Bruno Laganá \\ Università degli Studi Mediterranea di \\ Reggio Calabria, Italy \\ Email: giuseppe.ruggeri@unirc.it, \\ lagana.bruno@gmail.com
}

\begin{abstract}
The Minibus public transportation sector and road safety remains a significant challenge in Africa. We propose a low cost system to monitor the taxi industry and encourage safe driving. A low cost capacitive proximity sensor for seat occupancy detection based on the loading mode capacitive sensing technique is designed. The capacitive sensor uses a single electrode to detect an occupant. We use ZigBee modules for a dynamic wireless system integration where sensors can be added or removed without modifications. A mathematical model of the capacitive sensor is developed and we determine the capacitance on the sensor's electrode. The occupied capacitance is double the unoccupied capacitance. Our results show that the proposed capacitive sensor can distinguish clearly between an unoccupied and occupied seat.
\end{abstract}

\section{INTRODUCTION}

Road-based public transportation has come to mean something unique in the context of South Africa in particular and sub-Saharan Africa in general. This sector has originally evolved to meet the demand of human mobility, but unfortunately a crucial demand that has fallen victim to the incentive of personal wealth, is personal safety. What makes this tragedy especially unjust is who the victims are: the poor have few other options due to the inadequate public transport network in sub-Saharan Africa. The damage is not limited to "the bodies of loved ones scattered on our roads" (Arrive Alive Campaign on taxi deaths [1]). It extends to other vehicles involved in minibus accidents, and more broadly affects the economy through the large number of employees and breadwinners lost in the senseless carnage.

The World Health Organization (WHO) African Region [2] has some of the world's highest road traffic fatalities globally with more than 32 deaths per 100,000 population annually making it the 9th leading cause of death in the region. The African region has less than $2 \%$ of the world's registered vehicles, but almost $20 \%$ of the global traffic deaths. An $80 \%$ increase in traffic deaths between 2000 and 2020 is predicted in [3].

In South Africa, the Minibus taxi sector dominates the informal public transportation system and has grown enormously in the past 20 years. Not only is it the most available mode

This work has been carried out under the framework of the international research project "PALMARES: an Internet of Smart Objects", funded by the Italian government within the Cooperlink initiative.

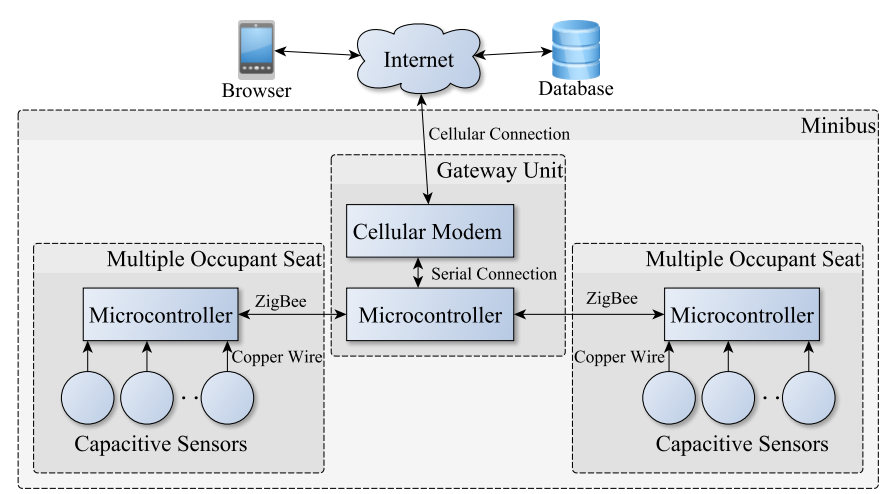

Fig. 1. The capacitive sensor integrated with the system. An occupant is detected and the information transmitted via ZigBee to the Gateway unit. From there the data is transmitted via a cellular carrier service and stored on a database.

of transport, but also the most affordable. In 2008 it held $67.9 \%$ of the collective transport market share [4]. According to the March 2011 Road Traffic Report [5], there are 285,858 registered Minibuses on the road. 1,408 of the Minibuses were involved in Fatal Crashes during 2010-11 with 1,795 fatalities. $57 \%$ of that number was passenger fatalities and $30 \%$ pedestrian fatalities.

The work in [6] and [7] have found that being monitored leads to safer driving behaviour. Seven key characteristics of a well performing urban transport system were identified by [8], with affordability and safety being the first two characteristics. This project therefore aims to develop a low cost system to monitor the taxi industry and encourage safe driving.

In the first stage of the project a sensing and reporting system is developed. This system consists of external sensors, retrofitted to the vehicle, which monitors the vehicle's speed, location, erratic driving and occupancy. The sensors include a GPS, accelerometer and seat-mounted capacitive proximity sensors. The collected data are transmitted real time, via a cellular carrier service, to a central database. It is then made available graphically on a mobile phone application.

In this paper, we present the design of a low cost capacitive proximity sensor for seat occupancy detection. In Section II a background is given of previous and related work done 
on capacitive sensors for occupancy detection. Section III discusses how the capacitive sensor work and how the network is set up with ZigBee. A mathematical model of the capacitive sensors and human interaction is determined in Section IV with the empirical results and conclusion following in Section $\mathrm{V}$ and Section VI.

\section{RELATED WORK}

Capacitive sensing describes a number of techniques to measure the capacitance between an electrode and its surroundings [9]. Capacitive sensing technology to detect human presence has proven to be a robust and reliable measurement technology with low power consumption that is ideal for the dynamic automotive environment. A number of studies have therefore gone into capacitive sensors for seat occupancy detection with several prototypes developed. Most of these occupancy detection sensors are developed for smart airbag systems [10], [11], [12] and to monitor drivers' vital signs [13], [14]. The focus of the above mentioned sensors are performance (fast response time) and high sensitivity. The work in [15] and [16] define three different capacitive sensing techniques: transmit mode, shunt mode and loading mode. The above referenced sensors use the shunt mode technique. In shunt mode, the body effectively becomes grounded, and screens the field, thereby reducing the current measured at the receiver electrode. According to [15] this technique cannot distinguish between a large mass far away and a small mass nearby, but multiple transmitter or receiver electrodes will break this degeneracy. Transmit mode is where the body acts as a virtual extension of the transmitter to the receiver electrode, which is not practical for occupancy detection. Loading mode capacitive sensing measures the change in capacitance between a single electrode and ground.

Transmit and shunt mode capacitive sensing techniques therefore use multiple electrodes installed on the sitting area and backrest for detection of a single occupant. Since this setup only detects a single occupant, the complexity of multi seat vehicles such as Minibus taxis with 14 seats would be high. The prototype in [10] uses 12 electrodes per seat, thus 168 electrodes would be required for a 14-seat Minibus taxi. This sensor system setup is therefore complex and a large number of copper wires are needed to connect all the electrodes with a microcontroller. The distribution of the sensors in the vehicle will cause difficulties when connecting the sensors' microcontrollers with a central microcontroller (collecting all the data) and even more copper wires will be required which increases cost and complexity. We have therefore found that a multiple occupant detection system has two shortcomings when considered for the Minibus industry, namely complexity and cost.

\section{CApacitive Sensing}

The presented occupancy detection design consists of capacitive sensors using the loading mode sensing technique (referred to as capacitive sensing throughout the rest of the paper) and ZigBee-enabled microcontrollers, as illustrated in

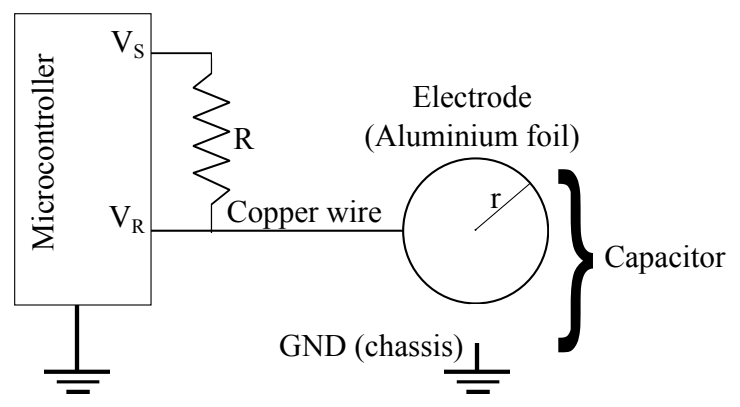

Fig. 2. Prototype model of the capacitive sensor. $V_{S}$ and $V_{R}$ are the Stimulus and Response pin respectively, $R$ is the $20 M \Omega$ resistor and $r$ the radius of the household aluminium foil $(0.1 \mathrm{~m})$. The sensor components are connected to the microcontroller via standard copper wires.

Fig. 1. Processing is done locally, which means processed data can be transmitted. The ZigBee network enables multiple sensor configurations to be connected wirelessly with a central microcontroller.

\section{A. Capacitive Sensor}

Capacitance is defined as the amount of electrical charge, which changes with voltage, stored between two nonconnected conductive plates. The capacitance depends primarily on the dielectric substrate (material between the two conductive plates) of the objects, the size of the plates and the distance between them.

The capacitive sensor set up can be seen in Fig. 2. The low cost setup of the system requires a high value resistor $(R)$ between the stimulus pin and the response pin. The high value resistor $(10 M \Omega-50 M \Omega)$ ensures a sufficient charge/discharge time in the order of $500 \mu \mathrm{s}$. A higher $R$ will result in a more sensitive capacitive sensor, but with more noise. The electrode is the sensor component to detect human proximity and is connected via a standard copper wire to the response pin. The sensor electrode is built up from a thin circular metal plate covered by a piece of non-conducting material. We created the electrode using household aluminium. The circular form decreases stray capacitance that forms around the edges [17].

Two digital pins are setup to be in one of two modes; the stimulus pin, which changes to a new state and waits for the response pin to change to the same state as the stimulus pin. The following sequence describes how the capacitive sensor is measured:

1) The stimulus pin is set as output and response pin as input.

2) A timer is started and the stimulus pin is set to logical high $\left(V_{C C}\right)$ which charges the capacitor until the response pin crosses the threshold logical high $\left(V_{I H}\right)$. The timer is then momentarily stopped (paused) while the response pin charges completely to $V_{C C}$.

3) The timer is resumed when the stimulus pin is set to logical low $\left(V_{O L}\right)$ which discharges the capacitor until the response pin crosses the threshold logical low $\left(V_{I L}\right)$.

4) The timer determines the time it takes for the response pin to charge to the logical opposite and resets to zero 


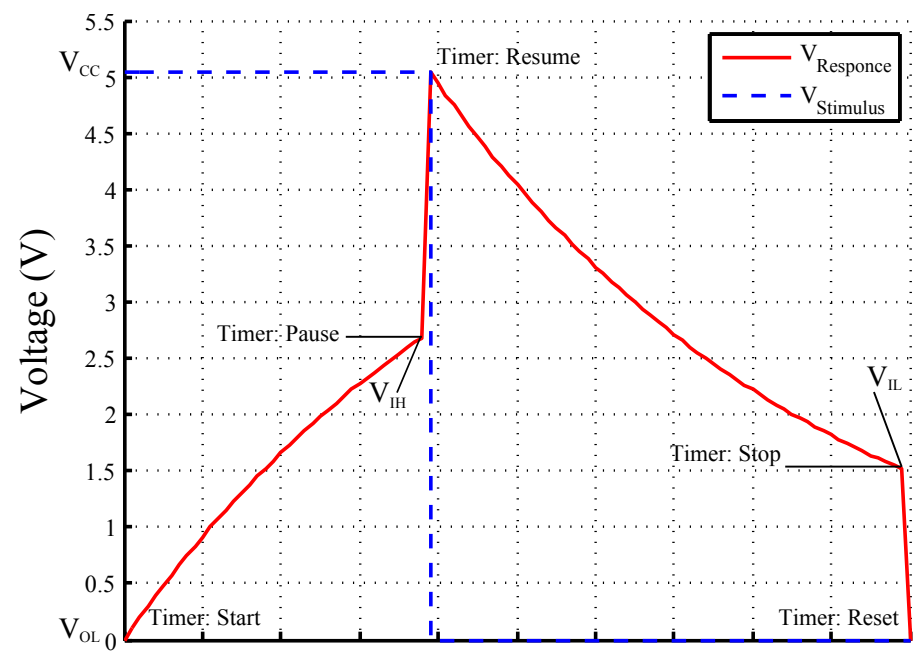

Fig. 3. One cycle of the capacitive sensor's charge/discharge plot. The plot has a $V_{C C}$ of $5.04 \mathrm{~V}$ with $V_{I H}$ and $V_{I L}$ measured as $2.64 \mathrm{~V}$ and $1.51 \mathrm{~V}$, respectively.

each time the stimulus pin changes to logical high. The change in capacitance can then be determined from the timer value before each reset occurring at the end of a cycle.

5) The process is repeated.

The above described sequence can be seen in the capacitive sensor's charge/discharge plot in Fig. 3. The value of the timer is directly proportional to $\tau$, the $R C$ time-constant (1). Since the resistor value $(R)$ stays constant, the delay depends solely on the capacitance $(C)$.

$$
\tau=R \times C
$$

When a human body enters the proximity of the sensor, the electrical properties between the sensor electrode and ground will increases the total capacitance resulting in a higher $R C$ time-constant. This will increase the capacitor charge/discharge time. It is therefore possible to determine if a seat is occupied by interpreting the timer value. The software is set up to automatically accommodate for external variance by adjusting the detection threshold. A thorough description of the mathematical model follows in Section IV.

\section{B. Sensor Network}

A ZigBee network (based on the IEEE 802.15.4 networking protocol) is used to provide wireless connectivity between the sensors' microcontrollers and the Gateway unit (see Fig. 1). This enables sensors to be added or removed from the system without modifying or updating the wiring or Gateway unit. Accurate data transmission is ensured since ZigBee has built in data-packet building and employs Carrier Sense Multiple Access with Collision Avoidance (CSMA/CA) to avoid packet collisions. The topology of the network is a star arrangement [18]. In this configuration the Gateway unit acts as a coordinator at the centre, with end devices connected around it and the end devices do not communicate with each other directly.

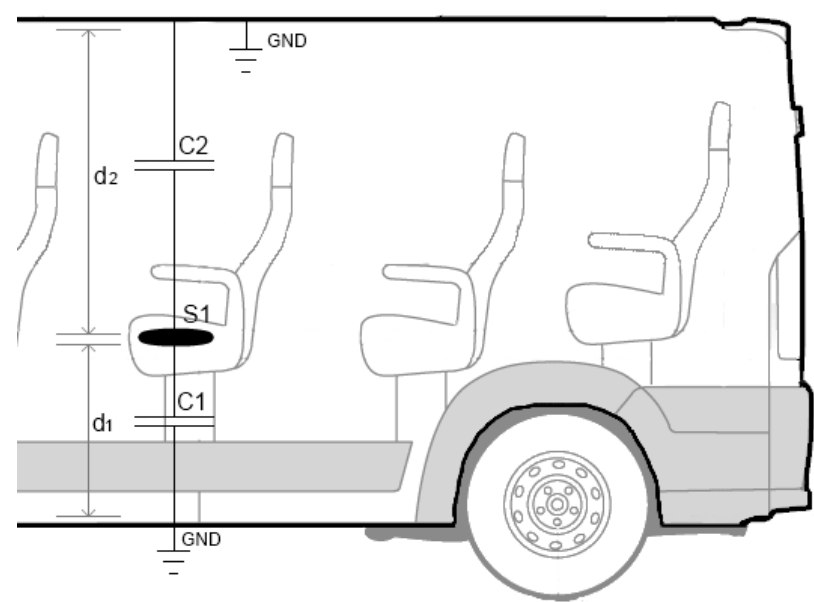

Fig. 4. Simplified model of the capacitance between the sensor plate and Minibus Taxi. $S_{1}$ represents a single capacitive seat sensor and $C_{1}$ and $C_{2}$ the capacitance between the sensor and chassis (at common ground). $C_{1}$ stays constant where $C_{2}$ changes according to the presence and size of an occupant. $d_{1}$ and $d_{2}$ are the distance between the sensor and the chassis.

Individual sensors in the integrated sensor system can also be identified and monitored independently.

Arduino UNOs are used as sensor microcontrollers and an Arduino Mega 2560 is used in the Gateway unit. The microcontrollers are equipped with XBee modules [19], to facilitate the ZigBee network. The Arduino microcontroller used in the Gateway unit is serially connected with a Sierra Wireless modem, which provides GPRS access into the database and visualization platform. The GPRS modem transmits processed occupancy and mobility information.

\section{Simplified Mathematical Model of a CAPaCitive SEnsor in a Minibus}

In this section, we develop a simplified mathematical model of the capacitive sensor in a vehicle. Our analysis of the Minibus taxi and capacitive sensors has indicated that a large number of factors can influence the sensors' capacitance. The purpose of the model of the capacitive sensor is to determine the relationship between the capacitance of an unoccupied and occupied seat. The simplified model can be seen in Fig. 4 with its corresponding circuit diagram in Fig. 5.

The Minibus chassis was chosen as the common ground (GND) and reference point for the capacitive sensors. As explained in Section III a capacitor is any arrangement of two conductors separated by an electric insulator called the dielectric substrate. The sensor's electrode with respect to the chassis (GND) with the non-conducting areas in between therefore forms a capacitor. We approximate this configuration as a parallel plate capacitor model assuming that the charge density on the plates are uniformly distributed and that the only capacitance exists between the sensor and the chassis as indicated in Fig. 4.

The capacitance of two plates in parallel is given by

$$
C=\frac{\epsilon_{o} \epsilon_{r} A}{d}
$$




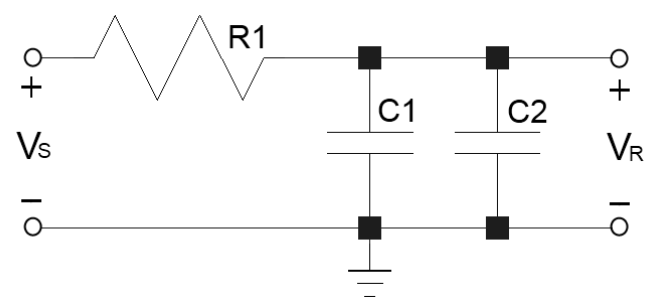

Fig. 5. Circuit representation of the capacitive seat sensor. $V_{S}$ is the send pin and $V_{R}$ the receive pin.

with:

$A$ plate area $\left[\mathrm{m}^{2}\right]$

$d \quad$ distance between plates $[\mathrm{m}]$

$\epsilon_{o} \quad$ permittivity of free space $=8.854 \times 10^{-12} \mathrm{~F} / \mathrm{m}$

$\epsilon_{r} \quad$ relative permittivity of the dielectric substrate between the plates [dimensionless]

Assuming that $A$ and $d$ stays constant and since $\epsilon_{o}$ is a constant, only $\epsilon_{r}$ can change the capacitance.

With two parallel plates forming a capacitor the electric field lines are not limited to the sensor, but curves outside the electrode causing the capacitance to be higher than what we would have calculated from the ideal expression (2). This effect is known as fringing. The capacitive sensor's electrode size $(A)$ is therefore increased, for calculations, by $13 \%$ to accommodate the fringing effect [17].

In Fig. 4, $S_{1}$ represents a single capacitive sensor where $C_{1}$ and $C_{2}$ are the parallel plate capacitance with respect to GND. Each of the seats will have such a sensor and thus a similar capacitive model. From Fig. 5, $V_{S}$ is the Stimulus pin's output voltage, $V_{R}$ is the Response pin's input voltage, and $R$ is the constant resistor. $C_{2}$ is the capacitance which changes with the presence of a human body.

Since the dielectric substrate consists partly of air, an effective dielectric constant is derived by approximating the capacitive sensor as a microstrip transmission line. Microstrip transmission lines consist of a conductive strip of width ' $W$ ' (capacitive sensor's electrode diameter of $0.2 \mathrm{~m}$ ) and a wider ground plane (vehicle's chassis), separated by a dielectric substrate of thickness ' $H$ ' (area between electrode and ground). The upper dielectric substrate is typically air. The effective dielectric constant $\left(\epsilon_{e}\right)$ is somewhat less that the substrate's dielectric constant since a part consists of air and can be approximated, according to [20],with the following expression:

$$
\begin{aligned}
& \text { when }\left(\frac{W}{H}\right)<1 \\
& \qquad \begin{aligned}
\epsilon_{e}=\frac{\epsilon_{r}+1}{2} & +\frac{\epsilon_{r}-1}{2}\left[\left(1+12\left(\frac{H}{W}\right)\right)^{\frac{-1}{2}}\right. \\
& \left.+0.04\left(1-\left(\frac{W}{H}\right)\right)^{2}\right]
\end{aligned}
\end{aligned}
$$

$$
\begin{aligned}
& \text { when }\left(\frac{W}{H}\right) \geq 1 \\
& \qquad \epsilon_{e}=\frac{\epsilon_{r}+1}{2}+\frac{\epsilon_{r}-1}{2}\left(1+12\left(\frac{H}{W}\right)\right)^{\frac{-1}{2}}
\end{aligned}
$$

As explained in Section III, the time constant depends solely on $C_{T O T A L}$ and we know from [21] that the following expression represents two capacitors in parallel in a circuit:

$$
C_{\text {TOTAL }}=C_{1}+C_{2}
$$

$C_{1}$ stays constant where $C_{2}$ can be in either one of two states; occupied (OCC), or unoccupied (UNOCC). In the case of an unoccupied state, the total capacitance will be:

$$
C_{T O T A L(U N O C C)}=\epsilon_{o} A\left(\frac{d_{1} \epsilon_{e 2_{U N O C C}}+d_{2} \epsilon_{e 1}}{d_{1} d_{2}}\right)
$$

where $\epsilon_{e 2_{U N O C C}}$ and $\epsilon_{e 1}$ is the effective dielectric constant (from (4)) of respectively the dielectric substrate above and below the sensor electrode. Similarly, the total capacitance when occupied is expressed as:

$$
C_{T O T A L(O C C)}=\epsilon_{o} A\left(\frac{d_{1} \epsilon_{e 2 O C C}+d_{2} \epsilon_{e 1}}{d_{1} d_{2}}\right) .
$$

where $\epsilon_{e 2 O C C}$ is the effective dielectric constant (from (3)) of the substrate above the sensor electrode when an occupant is present.

We approximate the vehicle seat's $\epsilon_{r}$ as 2.73 since the main composition of vehicle seat material are polyethylene foam ( $\epsilon_{r}$ of 2.26) and rubber foam ( $\epsilon_{r}$ of 3.20) [22]. From (4) we calculated $\epsilon_{e 1}$ as 2.19 since the capacitive sensor electrode has a diameter (' $W$ ') of $0.20 \mathrm{~m}$ and estimated dielectric substrate height (' $\left.H^{\prime}\right)$ of $0.10 m\left(\frac{W}{H}>1\right)$. The reason for this low substrate height is because of the metal found in the seat which effectively reduces the distance between the sensor electrode and ground. Similarly $\epsilon_{e 2_{U N O C C}}$ is estimated as 1.23 , with a dielectric constant of 1.30 (cotton) and dielectric substrate height of $0.05 m\left(\frac{W}{H}>1\right)$.

An occupant on the seat will increase $\epsilon_{r}$ substantially since the human body consists $70.4 \%$ of water [23] with an $\epsilon_{r}$ of 74.1 [24]. We therefore approximate the human body with the same dielectric properties of water and calculate $\epsilon_{e 2}$ as 42.59 from (3), with ' $W$ ' and ' $H$ ' as $0.2 m$ and $1.4 m$ respectively $\left(\frac{W}{H}<1\right)$. The inside height of the vehicle for the experimental setup were determined as $0.1 \mathrm{~m}$ for $d_{1}$ and $1.4 \mathrm{~m}$ for $d_{2}$.

The theoretical model is concluded by calculating the approximated capacitance on the sensor electrode. The unoccupied capacitance is calculated from (6) as $15.82 p F$ and the occupied capacitance from (7) as $36.41 p F$, with $A=$ $\pi(0.1 \times 1.13)^{2}$ because of the fringing effect. 


\section{ExPerimental Setup and Results}

The experimental setup consists of two microcontrollers, each one equiped with a ZigBee module and three aluminium electrodes. Each of the three sensors' charge time (in microseconds) were determined and logged with 6000 samples taken. The following table shows the average of each of the sensors' unoccupied and occupied charge times.

TABLE I

CHARGE TIME OF THREE CAPACITIVE SENSORS.

\begin{tabular}{|c|c|c|}
\hline & Unoccupied $(\mu s)$ & Occupied $(\mu s)$ \\
\hline Sensor 1 & 309 & 652 \\
\hline Sensor 2 & 316 & 664 \\
\hline Sensor 3 & 382 & 750 \\
\hline Average & 336 & 689 \\
\hline
\end{tabular}

The empirical capacitance is determined from the following equation:

$$
C=\frac{-t}{R \ln \left(1-\frac{V_{R}}{V_{S}}\right)}
$$

with $R=20 M \Omega, V_{S}=5.04 \mathrm{~V}$ and $V_{R}=2.64 \mathrm{~V}$. The capacitance can be calculated from (8) as $22.62 p F$ for $C_{U N O C C}$ and $46.41 \mathrm{pF}$ for $C_{O C C}$ taking the average charge time from Table I. The $\frac{C_{U N O C C}}{C O C C}$ ratio is determined as 2.05 . The theoretical and empirical results are summarised in Fig. 6. The empirical occupied capacitance is that of a medium size occupant. Two additional measurements of occupants with different sizes are included in the figure, the capacitance on the sensor electrode with a small occupant present and the capacitance with a large occupant present.

The relationship of the unoccupied and the occupied capacitance is therefore approximated by:

$$
C_{O C C} \approx 2 C_{U N O C C}
$$

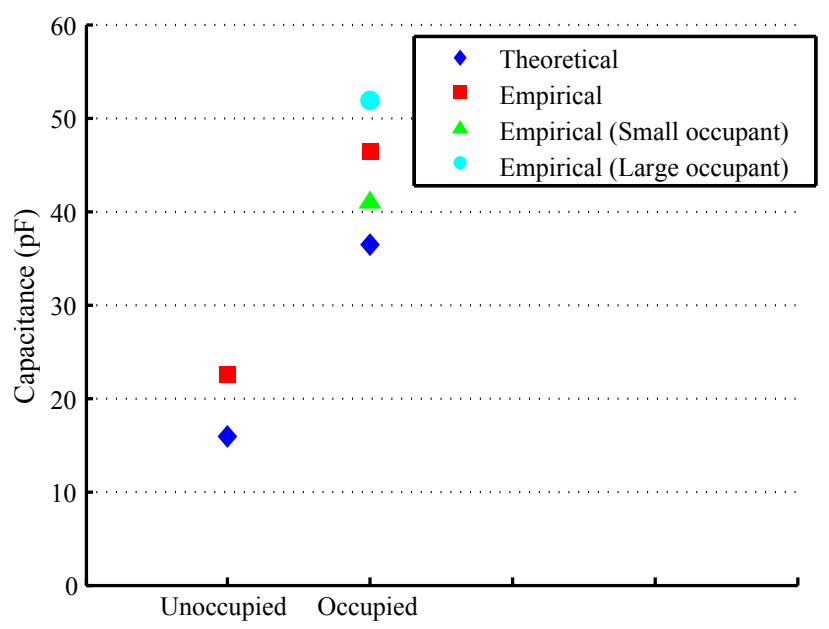

Fig. 6. Summary of the theoretical and empirical capacitance on the electrode.

By comparing the theoretical and empirical results, a $30.1 \%$ difference is found between the unoccupied capacitance and $21.5 \%$ difference between the occupied capacitance.

\section{CONCLUSION}

From the theoretical and empirical results, we have determined that this loading mode capacitive sensing technique can distinguish clearly between an occupied and unoccupied seat. The relationship between unoccuied and occuied is approximatly double. The simple and low cost capacitive sensor system is ideal for occupancy detection in multiple seat vehicles, because of its low cost design and wireless system integration. Compared to other occupant detection systems, this simple electrode and wireless ZigBee module configuration reduces wiring complexity, maintenance, cost and ensures greater flexibility for instalment within the vehicle.

\section{REFERENCES}

[1] Arrive Alive. (2008, Sep.) Minibus taxis and road safety. [Online]. Available: www.arrivealive.co.za/pages.aspx $? \mathrm{i}=2850$

[2] World Health Organization, "Status report on road safety in countries of the who african region," 2009.

[3] E. Kopits and M. Cropper, "Traffic fatalities and economic growth," Accident Analysis \& Prevention, vol. 37, no. 1, pp. 169-178, 2005.

[4] Trans-Africa Consortium, "Overview of public transport in sub-saharan africa," 2008.

[5] Road Traffic Management Corporation, "Road traffic report 2010/2011," March 2011.

[6] J. Hickman and E. Geller, "Self-management to increase safe driving among short-haul truck drivers," Journal of Organizational Behavior Management, vol. 23, no. 4, pp. 1-20, 2005.

[7] D. Johnson and M. Trivedi, "Driving style recognition using a smartphone as a sensor platform," in Intelligent Transportation Systems (ITSC), 2011 14th International IEEE Conference on, oct. 2011, pp. $1609-1615$.

[8] A. Smith, "A study of institutional, financial and regulatory frameworks of urban transport in large sub-saharan cities," SSATP Working Paper No. 82, Jul 2005, africa Region The World Bank.

[9] R. Wimmer, "Capacitive sensors for whole body interaction," Whole Body Interaction, pp. 121-133, 2011.

[10] B. George, H. Zangl, T. Bretterklieber, and G. Brasseur, "Seat occupancy detection based on capacitive sensing," Instrumentation and Measurement, IEEE Transactions on, vol. 58, no. 5, pp. 1487 -1494, may 2009.

[11] H. Zangl, T. Bretterklieber, D. Hammerschmidt, and T. Werth, "Seat occupancy detection using capacitive sensing technology," SAE Technical Paper, pp. 01-0908, 2008.

[12] A. Satz, D. Hammerschmidt, and D. Tumpold, "Capacitive passenger detection utilizing dielectric dispersion in human tissues," Sensors and Actuators A: Physical, vol. 152, no. 1, pp. 1-4, 2009.

[13] K. Kim, Y. Lim, and K. Park, "Common mode noise cancellation for electrically non-contact ecg measurement system on a chair," in Engineering in Medicine and Biology Society, 2005. IEEE-EMBS 2005. 27th Annual International Conference of the. IEEE, 2006, pp. 58815883.

[14] S. Jung, H. Shin, and W. Chung, "Highly sensitive driver health condition monitoring system using nonintrusive active electrodes," Sensors and Actuators B: Chemical, 2012.

[15] J. Paradiso and N. Gershenfeld, "Musical applications of electric field sensing," Computer music journal, vol. 21, no. 2, pp. 69-89, 1997.

[16] J. Smith, "Electric field imaging," Ph.D. dissertation, Massachusetts Institute of Technology, 1998.

[17] E. Hoch, "Electrode effects in the measurement of power factor and dielectric constant of sheet insulating materials," Bell System Technical Journal, vol. 5, p. 555, 1926.

[18] R. Faludi, Building Wireless Sensor Networks: With ZigBee, XBee, Arduino, and Processing. O'Reilly Media, Inc., 2010.

[19] Digi International Inc. (2012, Sep.) XBee ZB Module. [Online]. Available: http://www.digi.com/products/wireless-wired-embeddedsolutions/zigbee-rf-modules/zigbee-mesh-module/xbee-zb-module

[20] I. Bahl and D. Trivedi, "A designer's guide to microstrip line," Microwaves, vol. 16, no. 5, pp. $174-176,1977$.

[21] B. Guru and H. Hızıroğlu, Electromagnetic field theory fundamentals. Cambridge Univ Pr, 2004. 
[22] G. Elert. (2012, Aug.) The Physics Hypertextbook - Dielectrics. [Online]. Available: http://physics.info/dielectrics/

[23] P. Watson, I. Watson, and R. Batt, "Total body water volumes for adult males and females estimated from simple anthropometric measurements," The American journal of clinical nutrition, vol. 33, no. 1, pp. 27-39, 1980.

[24] S. Gabriel, R. Lau, and C. Gabriel, "The dielectric properties of biological tissues: III. Parametric models for the dielectric spectrum of tissues," Physics in medicine and biology, vol. 41, p. 2271, 1996. 